\title{
The necessary and sufficient conditions
} for the existence of a kind of Hilbert-type multiple integral inequality with the non-homogeneous kernel and its applications

\author{
Yong Hong ${ }^{1}$, Qiliang Huang ${ }^{2 *}$, Bicheng Yang ${ }^{2}$ and Jianquan Liao ${ }^{2}$
}

"Correspondence:

qlhuang@yeah.net

${ }^{2}$ Department of Mathematics,

Guangdong University of

Education, Guangzhou, Guangdong

510303, P.R. China

Full list of author information is

available at the end of the article

\begin{abstract}
For $x=\left(x_{1}, \ldots, x_{n}\right), u(x)=\left(\sum_{i=1}^{n} a_{i} x_{i}^{\rho}\right)^{1 / \rho}, v(y)=\left(\sum_{i=1}^{n} b_{i} y_{i}^{\rho}\right)^{1 / \rho}$, by using the methods and techniques of real analysis, the sufficient and necessary conditions for the existence of the Hilbert-type multiple integral inequality with the kernel $K(u(x), v(y))=G\left(u^{\lambda_{1}}(x) v^{\lambda_{2}}(y)\right)$ and the best possible constant factor are discussed. Furthermore, its application in the operator theory is considered.
\end{abstract}

MSC: 26D15;47A07

Keywords: Hilbert-type inequality; non-homogeneous kernel; sufficient and necessary conditions; best possible constant factor; bounded operator; operator norm

\section{Introduction}

For $n \geq 1, R_{+}^{n}=\left\{x=\left(x_{1}, \ldots, x_{n}\right): x_{i}>0, i=1, \ldots, n\right\}, a_{i}, b_{i}>0(i=1, \ldots, n), \omega(x)>0\left(x \in R_{+}^{n}\right)$, and $\rho>0$, we set

$$
\begin{aligned}
& u(x)=\left(\sum_{i=1}^{n} a_{i} x_{i}^{\rho}\right)^{\frac{1}{\rho}}, \quad v(y)=\left(\sum_{i=1}^{n} b_{i} y_{i}^{\rho}\right)^{\frac{1}{\rho}}, \\
& L_{\omega}^{p}\left(R_{+}^{n}\right):=\left\{f(x) \geq 0:\|f\|_{p, \omega}=\left(\int_{R_{+}^{n}} \omega(x) f^{p}(x) d x\right)^{1 / p}<+\infty\right\} .
\end{aligned}
$$

If $p>1, \frac{1}{p}+\frac{1}{q}=1, K(u, v) \geq 0(u, v>0)$, then the Hilbert-type multiple integral inequality is of the form

$$
\int_{R_{+}^{n}} \int_{R_{+}^{n}} K(u(x), v(y)) f(x) g(y) d x d y \leq M\|f\|_{p, u^{\alpha}}\|g\|_{q, v^{\beta}} .
$$

\section{Springer}


Define a singular integral operator $T$ :

$$
T(f)(y):=\int_{R_{+}^{n}} K(u(x), v(y)) f(x) d x, \quad y \in R_{+}^{n},
$$

then (1) may be rewritten as follows:

$$
\int_{R_{+}^{n}} T(f)(y) g(y) d y \leq M\|f\|_{p, u^{\alpha}}\|g\|_{q, v^{\beta}} .
$$

It is easy to prove that (1) is equivalent to the following inequality:

$$
\|T(f)\|_{p, v^{\nu}} \leq M\|f\|_{p, u^{\alpha}}\|g\|_{q, v^{\beta}}
$$

where $\gamma=\beta(1-p)$. When the operator $T$ satisfies (3), $T$ is called bounded operator from $L_{u^{\alpha}}^{p}\left(R_{+}^{n}\right)$ to $L_{v^{\gamma}}^{p}\left(R_{+}^{n}\right)$.

At present, there are lots of research results on Hilbert-type single integral inequality (cf. [1-14]). But there are relatively few studies on Hilbert-type multiple integral inequality. In particular, there are fewer studies on the necessary and sufficient conditions for the existence of the multiple integral inequality.

In this article, by using the methods and techniques of real analysis, we give the sufficient and necessary conditions for the existence of the Hilbert-type multiple integral inequality with the non-homogeneous kernel

$$
K(u(x), v(y))=G\left(u^{\lambda_{1}}(x) v^{\lambda_{2}}(y)\right),
$$

and calculate the best possible constant factor. Furthermore, its application in the operator theory is considered.

\section{Some lemmas}

Lemma 1 Suppose that $p>1, \frac{1}{p}+\frac{1}{q}=1, n \geq 1, \rho>0, \lambda_{1} \lambda_{2}>0, a_{i}, b_{i}>0(i=1, \ldots, n)$,

$$
\begin{aligned}
u(x)=\left(\sum_{i=1}^{n} a_{i} x_{i}^{\rho}\right)^{\frac{1}{\rho}}, v(y)=\left(\sum_{i=1}^{n} b_{i} y_{i}^{\rho}\right)^{\frac{1}{\rho}} . \\
\text { If } K(u(x), v(y))=G\left(u^{\lambda_{1}}(x) v^{\lambda_{2}}(y)\right) \text { is a non-negative measurable function, setting } \\
W_{1}:=\int_{R_{+}^{n}}(v(t))^{-\frac{\beta+n}{q}} K(1, v(t)) d t, \\
W_{2}:=\int_{R_{+}^{n}}(u(t))^{-\frac{\alpha+n}{p}} K(u(t), 1) d t,
\end{aligned}
$$

we have the following:

$$
\begin{aligned}
& \omega_{1}(x):=\int_{R_{+}^{n}}(v(y))^{-\frac{\beta+n}{q}} K(u(x), v(y)) d y=(u(x))^{\frac{\lambda_{1}}{\lambda_{2}}\left(\frac{\beta+n}{q}-n\right)} W_{1}, \\
& \omega_{2}(x):=\int_{R_{+}^{n}}(u(x))^{-\frac{\alpha+n}{p}} K(u(x), v(y)) d x=(v(y))^{\frac{\lambda_{2}}{\lambda_{1}}\left(\frac{\alpha+n}{p}-n\right)} W_{2} .
\end{aligned}
$$


Proof Since $v(a y)=a v(y)(a>0)$, in view of $K(t u, v)=K\left(u, t^{\frac{\lambda_{1}}{\lambda_{2}}} v\right)$, setting $t=u^{\frac{\lambda_{1}}{\lambda_{2}}}(x) y$, we find $d y=u^{\frac{-n \lambda_{1}}{\lambda_{2}}}(x) d t$ and

$$
\begin{aligned}
\omega_{1}(x) & =\int_{R_{+}^{n}}(v(y))^{-\frac{\beta+n}{q}} K\left(1, u^{\frac{\lambda_{1}}{\lambda_{2}}}(x) v(y)\right) d y \\
& =\int_{R_{+}^{n}}\left(u^{-\frac{\lambda_{1}}{\lambda_{2}}}(x) v(t)\right)^{-\frac{\beta+n}{q}} K(1, v(t)) u^{\frac{-n \lambda_{1}}{\lambda_{2}}}(x) d t \\
& =(u(x))^{\frac{\lambda_{1}}{\lambda_{2}}\left(\frac{\beta+n}{q}-n\right)} W_{1} .
\end{aligned}
$$

In the same way, we have

$$
\omega_{2}(x)=(v(y))^{\frac{\lambda_{2}}{\lambda_{1}}\left(\frac{\alpha+n}{p}-n\right)} W_{2} .
$$

The lemma is proved.

Lemma 2 (cf. [15]) If $p_{i}>0, a_{i}>0, \alpha_{i}>0(i=1, \ldots, n)$ and $\psi(t)$ is a measurable function, then we have the following:

$$
\begin{aligned}
\int & \cdots \int_{\left\{x_{i}>0 ; \sum_{i=1}^{n}\left(\frac{x_{i}}{a_{i}}\right)^{\alpha_{i}} \leq 1\right\}} \psi\left(\sum_{i=1}^{n}\left(\frac{x_{i}}{a_{i}}\right)^{\alpha_{i}}\right) x_{1}^{p_{1}-1} \cdots x_{n}^{p_{n}-1} d x_{1} \cdots d x_{n} \\
= & \frac{a_{1}^{p_{1}} \cdots a_{n}^{p_{n}} \Gamma\left(\frac{p_{1}}{\alpha_{1}}\right) \cdots \Gamma\left(\frac{p_{n}}{\alpha_{n}}\right)}{\alpha_{1} \cdots \alpha_{n} \Gamma\left(\sum_{i=1}^{n} \frac{p_{i}}{\alpha_{i}}\right)} \int_{0}^{1} \psi(t) t^{\sum_{i=1}^{n} \frac{p_{i}}{\alpha_{i}}-1} d t
\end{aligned}
$$

where $\Gamma(t)$ is the gamma function. In particular, for $\alpha_{i}=\rho, p_{i}=1, b_{i}=\frac{1}{a_{i}^{\rho}}(i=1, \ldots, n)$, we have

$$
\begin{gathered}
\int \cdots \int_{\left\{x_{i}>0 ; \sum_{i=1}^{n} b_{i} x_{i}^{\rho} \leq 1\right\}} \psi\left(\sum_{i=1}^{n} b_{i} x_{i}^{\rho}\right) d x_{1} \cdots d x_{n} \\
=\frac{\prod_{i=1}^{n} b_{i}^{-\frac{1}{\rho}} \Gamma^{n}\left(\frac{1}{\rho}\right)}{\rho^{n} \Gamma\left(\frac{n}{\rho}\right)} \int_{0}^{1} \psi(t) t^{\frac{n}{\rho}-1} d t .
\end{gathered}
$$

\section{Main results}

We set

$$
\begin{aligned}
& \Omega(a<b)=\left\{x=\left(x_{1}, \ldots, x_{n}\right) ; a<u(x)<b\right\}, \\
& \Omega^{\prime}(a<b)=\left\{x=\left(x_{1}, \ldots, x_{n}\right) ; a<v(y)<b\right\} .
\end{aligned}
$$

Theorem 1 Suppose that $n \geq 1, p>1, \frac{1}{p}+\frac{1}{q}=1, \rho>0, \alpha, \beta \in R, \lambda_{1} \lambda_{2}>0, a_{i}>0, b_{i}>0$ $(i=1, \ldots, n), u(x)=\left(\sum_{i=1}^{\infty} a_{i} x_{i}^{\rho}\right)^{1 / \rho}, v(y)=\left(\sum_{i=1}^{\infty} b_{i} y_{i}^{\rho}\right)^{1 / \rho}, K(u(x), v(y))=G\left(u^{\lambda_{1}}(x) v^{\lambda_{2}}(y)\right)$ is a non-negative measurable function,

$$
\begin{aligned}
& 0<W_{1}=\int_{R_{+}^{n}}(v(t))^{-\frac{\beta+n}{q}} K(1, v(t)) d t<\infty, \\
& 0<W_{2}=\int_{R_{+}^{n}}(u(t))^{-\frac{\alpha+n}{p}} K(u(t), 1) d t<\infty,
\end{aligned}
$$


and for $a=0, b=1($ or $a=1, b=+\infty)$,

$$
\begin{aligned}
& \int_{\Omega(a<b)}(v(t))^{-\frac{\beta+n}{q}} K(1, v(t)) d t>0, \\
& \int_{\Omega^{\prime}(a<b)}(u(t))^{-\frac{\alpha+n}{p}} K(u(t), 1) d t>0,
\end{aligned}
$$

then we have the following: There is a constant $M$ such that, for $f(x) \in L_{u^{\alpha}(x)}^{p}\left(R_{+}^{n}\right)$ and $g(y) \in$ $L_{v^{\gamma}(y)}^{p}\left(R_{+}^{n}\right)$, the following inequality

$$
\int_{R_{+}^{n}} \int_{R_{+}^{n}} K(u(x), v(y)) f(x) g(y) d x d y \leq M\|f\|_{p, u^{\rho}}\|g\|_{q, v^{\rho}}
$$

holds true if and only if the equality $\frac{n \lambda_{1}+\alpha \lambda_{2}}{p}=\frac{n \lambda_{2}+\beta \lambda_{1}}{q}$ is valid.

Proof We assume that (4) is valid and set $c=\frac{n \lambda_{2}+\beta \lambda_{1}}{q}-\frac{n \lambda_{1}+\alpha \lambda_{2}}{p}$.

(i) For $\lambda_{1}, \lambda_{2}>0$, if $c>0$, putting $\varepsilon>0$ small enough and

$$
\begin{aligned}
& f(x)= \begin{cases}(u(x))^{\left(-\alpha-n-\lambda_{1} \varepsilon\right) / p}, & u(x)>1, \\
0, & 0<u(x) \leq 1,\end{cases} \\
& g(y)= \begin{cases}(v(y))^{\left(-\beta-n+\lambda_{2} \varepsilon\right) / q}, & 0<v(y)<1, \\
0, & v(y) \geq 1,\end{cases}
\end{aligned}
$$

by Lemma 2 , we have

$$
\begin{aligned}
\|f\|_{p, u^{\rho}}\|g\|_{q, v^{\rho}} & \\
= & \left(\int_{\Omega(1<+\infty)}(u(x))^{-n-\lambda_{1} \varepsilon} d x\right)^{1 / p}\left(\int_{\Omega^{\prime}(0<1)}(v(y))^{-n+\lambda_{2} \varepsilon} d y\right)^{1 / q} \\
= & \left(\frac{\Gamma^{n}\left(\frac{1}{\rho}\right)}{a_{1}^{1 / \rho} \cdots a_{n}^{1 / \rho} \rho^{n-1} \Gamma\left(\frac{n}{\rho}\right)} \frac{1}{\lambda_{1} \varepsilon}\right)^{1 / p} \\
& \times\left(\frac{\Gamma^{n}\left(\frac{1}{\rho}\right)}{b_{1}^{1 / \rho} \cdots b_{n}^{1 / \rho} \rho^{n-1} \rho^{n-1} \Gamma\left(\frac{n}{\rho}\right)} \frac{1}{\lambda_{2} \varepsilon}\right)^{1 / q} \\
= & \frac{\Gamma^{n}\left(\frac{1}{\rho}\right)}{\lambda_{1}^{1 / p} \lambda_{2}^{1 / q} \rho^{n-1} \Gamma\left(\frac{n}{\rho}\right) \varepsilon}\left(\prod_{i=1}^{n} a_{i}^{-1 / \rho}\right)^{1 / p}\left(\prod_{i=1}^{n} b_{i}^{-1 / \rho}\right)^{1 / q}, \\
\int_{R_{+}^{n}} & \int_{R_{+}^{n}} K(u(x), v(y)) f(x) g(y) d x d y \\
= & \int_{\Omega(1<+\infty)}(u(x))^{\left(-\alpha-n-\lambda_{1} \varepsilon\right) / p}\left(\int_{\Omega^{\prime}(0<1)} K(u(x), v(y))(v(y))^{\left(-\beta-n+\lambda_{2} \varepsilon\right) / q} d y\right) d x \\
= & \int_{\Omega(1<+\infty)}(u(x))^{\left(-\alpha-n-\lambda_{1} \varepsilon\right) / p}\left(\int_{\Omega^{\prime}(0<1)} K\left(1, v\left(u^{\frac{\lambda_{1}}{\lambda_{2}}}(x) y\right)\right)(v(y))^{\left(-\beta-n+\lambda_{2} \varepsilon\right) / q} d y\right) d x \\
= & \int_{\Omega(1<+\infty)}(u(x))^{\left(-\alpha-n-\lambda_{1} \varepsilon\right) / p}\left(\int_{\Omega^{\prime}\left(0<u^{\frac{\lambda}{\lambda_{2}}}(x)\right)} K(1, v(t))\right.
\end{aligned}
$$




$$
\begin{aligned}
& \left.\times\left(u^{-\frac{\lambda_{1}}{\lambda_{2}}}(x) v(t)\right)^{\left(-\beta-n+\lambda_{2} \varepsilon\right) / q} u^{-\frac{n \lambda_{1}}{\lambda_{2}}}(x) d t\right) d x \\
= & \int_{\Omega(1<+\infty)}(u(x))^{-n+\frac{c}{\lambda_{2}}-\lambda_{1} \varepsilon}\left(\int_{\Omega^{\prime}\left(0<u^{\frac{\lambda_{1}}{\lambda_{2}}}(x)\right)} K(1, v(t))(v(t))^{\left(-\beta-n+\lambda_{2} \varepsilon\right) / q} d t\right) d x \\
\geq & \int_{\Omega(1<+\infty)}(u(x))^{-n+\frac{c}{\lambda_{2}}-\lambda_{1} \varepsilon} d x \int_{\Omega^{\prime}(0<1)} K(1, v(t))(v(t))^{\left(-\beta-n+\lambda_{2} \varepsilon\right) / q} d t .
\end{aligned}
$$

Hence, by (4), (5) and (6), we have the following:

$$
\begin{aligned}
& \int_{\Omega(1<+\infty)}(u(x))^{-n+\frac{c}{\lambda_{2}}-\lambda_{1} \varepsilon} d x \int_{\Omega^{\prime}(0<1)} K(1, v(t))(v(t))^{\left(-\beta-n+\lambda_{2} \varepsilon\right) / q} d t \\
& \quad \leq M \frac{\Gamma^{n}\left(\frac{1}{\rho}\right)}{\lambda_{1}^{1 / p} \lambda_{2}^{1 / q} \rho^{n-1} \Gamma\left(\frac{n}{\rho}\right) \varepsilon}\left(\prod_{i=1}^{n} a_{i}^{-1 / \rho}\right)^{1 / p}\left(\prod_{i=1}^{n} b_{i}^{-1 / \rho}\right)^{1 / q} .
\end{aligned}
$$

For $\lambda_{2}>0, c>0, \varepsilon>0$ small enough, $-n+\frac{c}{\lambda_{2}}-\lambda_{1} \varepsilon>-n$, it follows that $\int_{\Omega(1<+\infty)}(u(x))^{-n+\frac{c}{\lambda_{2}}-\lambda_{1} \varepsilon} d x=+\infty$, which contradicts inequality (7) in view of $\int_{\Omega^{\prime}(0<1)} K(1, v(t))(v(t))^{\left(-\beta-n+\lambda_{2} \varepsilon\right) / q} d t>0$. Hence it is not valid for $c>0$.

If $c<0$, putting $\varepsilon>0$ small enough and

$$
\begin{aligned}
& f(x)= \begin{cases}(u(x))^{\left(-\alpha-n+\lambda_{1} \varepsilon\right) / p}, & 0<u(x)<1, \\
0, & u(x) \geq 1,\end{cases} \\
& g(y)= \begin{cases}(v(y))^{\left(-\beta-n+\lambda_{2} \varepsilon\right) / q}, & v(y)>1, \\
0, & 0<v(y) \leq 1,\end{cases}
\end{aligned}
$$

in the same way, we have the following:

$$
\begin{aligned}
& \int_{\Omega^{\prime}(1<+\infty)}(v(y))^{-n-\frac{c}{\lambda_{1}}-\lambda_{2} \varepsilon} d y \int_{\Omega(0<1)} K(u(t), 1)(u(t))^{\left(-\alpha-n+\lambda_{1} \varepsilon\right) / p} d t \\
& \leq M \frac{\Gamma^{n}\left(\frac{1}{\rho}\right)}{\lambda_{1}^{\frac{1}{p}} \lambda_{2}^{\frac{1}{q}} \rho^{n-1} \Gamma\left(\frac{n}{\rho}\right) \varepsilon}\left(\prod_{i=1}^{n} a_{i}^{-\frac{1}{\rho}}\right)^{\frac{1}{p}}\left(\prod_{i=1}^{n} b_{i}^{-\frac{1}{\rho}}\right)^{\frac{1}{q}} .
\end{aligned}
$$

For $\lambda_{2}>0, c<0, \varepsilon>0$ small enough, hence $-n-\frac{c}{\lambda_{1}}-\lambda_{2} \varepsilon>-n$, it follows that $\int_{\Omega^{\prime}(1<+\infty)}(v(y))^{-n-\frac{c}{\lambda_{1}}-\lambda_{2} \varepsilon} d y=+\infty$, which contradicts inequality (8) in view of $\int_{\Omega(0<1)} K(u(t), 1)(u(t))^{\left(-\alpha-n+\lambda_{1} \varepsilon\right) / p} d t>0$. Hence, it is not valid for $c<0$.

Therefore, we prove that $c=0$, namely $\frac{n \lambda_{1}+\alpha \lambda_{2}}{p}=\frac{n \lambda_{2}+\beta \lambda_{1}}{q}$ is valid.

(ii) For $\lambda_{1}, \lambda_{2}<0$, we prove that $\frac{n \lambda_{1}+\alpha \lambda_{2}}{p}=\frac{p \lambda_{2}+\beta \lambda_{1}}{q}$ is valid as follows.

If $c>0$, putting $\varepsilon>0$ small enough and

$$
\begin{aligned}
& f(x)= \begin{cases}(u(x))^{\left(-\alpha-n-\lambda_{1} \varepsilon\right) / p}, & 0<u(x)<1, \\
0, & u(x) \geq 1,\end{cases} \\
& g(y)= \begin{cases}(v(y))^{\left(-\beta-n+\lambda_{2} \varepsilon\right) / q}, & v(y)>1, \\
0, & 0<v(y) \leq 1,\end{cases}
\end{aligned}
$$


Hong et al. Journal of Inequalities and Applications (2017) 2017:316

Page 6 of 12

we have

$$
\begin{aligned}
& \|f\|_{p, u^{\rho}}\|g\|_{q, v^{\rho}} \\
& =\left(\int_{\Omega(0<1)}(u(x))^{-n-\lambda_{1} \varepsilon} d x\right)^{1 / p}\left(\int_{\Omega^{\prime}(1<+\infty)}(v(y))^{-n+\lambda_{2} \varepsilon} d y\right)^{1 / q} \\
& =\frac{\Gamma^{n}\left(\frac{1}{\rho}\right)}{\left(-\lambda_{1}\right)^{1 / p}\left(-\lambda_{2}\right)^{1 / q} \rho^{n-1} \Gamma\left(\frac{n}{\rho}\right) \varepsilon}\left(\prod_{i=1}^{n} a_{i}^{-1 / \rho}\right)^{1 / p}\left(\prod_{i=1}^{n} b_{i}^{-1 / \rho}\right)^{1 / q}, \\
& \int_{R_{+}^{n}} \int_{R_{+}^{n}} K(u(x), v(y)) f(x) g(y) d x d y \\
& =\int_{\Omega(0<1)}(u(x))^{\left(-\alpha-n-\lambda_{1} \varepsilon\right) / p}\left(\int_{\Omega^{\prime}(1<+\infty)} K(u(x), v(y))(v(y))^{\left(-\beta-n+\lambda_{2} \varepsilon\right) / q} d y\right) d x \\
& =\int_{\Omega(0<1)}(u(x))^{\left(-\alpha-n-\lambda_{1} \varepsilon\right) / p}\left(\int_{\Omega^{\prime}\left(u^{\frac{\lambda_{1}}{\lambda_{2}}}(x)<+\infty\right)} K(1, v(t))\right. \\
& \left.\times\left(u^{-\frac{\lambda_{1}}{\lambda_{2}}}(x) v(t)\right)^{\left(-\beta-n+\lambda_{2} \varepsilon\right) / q} u^{-\frac{n \lambda_{1}}{\lambda_{2}}}(x) d t\right) d x \\
& =\int_{\Omega(0<1)}(u(x))^{-n+\frac{c}{\lambda_{2}}-\lambda_{1} \varepsilon}\left(\int_{\Omega^{\prime}\left(u^{\frac{\lambda_{1}}{\lambda_{2}}}(x)<+\infty\right)} K(1, v(t))(v(t))^{\left(-\beta-n+\lambda_{2} \varepsilon\right) / q} d t\right) d x \\
& \geq \int_{\Omega(0<1)}(u(x))^{-n+\frac{c}{\lambda_{2}}-\lambda_{1} \varepsilon} d x \int_{\Omega^{\prime}(1<+\infty)} K(1, v(t))(v(t))^{\left(-\beta-n+\lambda_{2} \varepsilon\right) / q} d t .
\end{aligned}
$$

Hence, by (4), (9) and (10), we have the following:

$$
\begin{gathered}
\int_{\Omega(0<1)}(u(x))^{-n+\frac{c}{\lambda_{2}}-\lambda_{1} \varepsilon} d x \int_{\Omega^{\prime}(1<+\infty)} K(1, v(t))(v(t))^{\left(-\beta-n+\lambda_{2} \varepsilon\right) / q} d t \\
\leq M \frac{\Gamma^{n}\left(\frac{1}{\rho}\right)}{\left(-\lambda_{1}\right)^{1 / p}\left(-\lambda_{2}\right)^{1 / q} \rho^{n-1} \Gamma\left(\frac{n}{\rho}\right) \varepsilon}\left(\prod_{i=1}^{n} a_{i}^{-1 / \rho}\right)^{1 / p}\left(\prod_{i=1}^{n} b_{i}^{-1 / \rho}\right)^{1 / q} .
\end{gathered}
$$

It is obvious that $\int_{\Omega(0<1)}(u(x))^{-n+\frac{c}{\lambda_{2}}-\lambda_{1} \varepsilon} d x=+\infty$, which contradicts inequality (11) in view of $\int_{\Omega^{\prime}(1<+\infty)} K(1, v(t))(v(t))^{\left(-\beta-n+\lambda_{2} \varepsilon\right) / q} d t>0$. Hence it is not valid for $c>0$.

If $c<0$, putting $\varepsilon>0$ small enough and

$$
\begin{aligned}
& f(x)= \begin{cases}(u(x))^{\left(-\alpha-n+\lambda_{1} \varepsilon\right) / p}, & u(x)>1, \\
0, & 0<u(x) \leq 1,\end{cases} \\
& g(y)= \begin{cases}(v(y))^{\left(-\beta-n-\lambda_{2} \varepsilon\right) / q}, & 0<v(y)<1, \\
0, & v(y) \geq 1,\end{cases}
\end{aligned}
$$

in the same way, we have

$$
\begin{aligned}
& \int_{\Omega^{\prime}(0<1)}(v(y))^{-n-\frac{c}{\lambda_{1}}-\lambda_{2} \varepsilon} d y \int_{\Omega(1<+\infty)} K(u(t), 1)(u(t))^{\left(-\alpha-n+\lambda_{1} \varepsilon\right) / p} d t \\
& \leq M \frac{\Gamma^{n}\left(\frac{1}{\rho}\right)}{\left(-\lambda_{1}\right)^{1 / p}\left(-\lambda_{2}\right)^{1 / q} \rho^{n-1} \Gamma\left(\frac{n}{\rho}\right) \varepsilon}\left(\prod_{i=1}^{n} a_{i}^{-1 / \rho}\right)^{1 / p}\left(\prod_{i=1}^{n} b_{i}^{-1 / \rho}\right)^{1 / q} .
\end{aligned}
$$


In virtue of $\int_{\Omega^{\prime}(0<1)}(v(y))^{-n-\frac{c}{\lambda_{1}}-\lambda_{2} \varepsilon} d y=+\infty$, (12) is a contradiction in view of $\int_{\Omega(1<+\infty)} K(u(t), 1)(u(t))^{\left(-\alpha-n+\lambda_{1} \varepsilon\right) / p} d t>0$. Hence, $c<0$ is not valid.

Therefore, we prove that $c=0$ is valid.

On the other hand, we assume that $\frac{n \lambda_{1}+\alpha \lambda_{2}}{p}=\frac{n \lambda_{2}+\beta \lambda_{1}}{q}$ is valid.

Setting $a=\frac{\alpha}{p q}+\frac{n}{p q}, b=\frac{\beta}{p q}+\frac{n}{p q}$, by Holder's inequality with weight and Lemma 1, we find

$$
\begin{aligned}
\int_{R_{+}^{n}} & \int_{R_{+}^{n}} K(u(x), v(y)) f(x) g(y) d x d y \\
= & \int_{R_{+}^{n}} \int_{R_{+}^{n}}\left(f(x) \frac{u^{a}(x)}{v^{b}(y)}\right)\left(g(y) \frac{v^{b}(y)}{u^{a}(x)}\right) K(u(x), v(y)) d x d y \\
\leq & \left(\int_{R_{+}^{n}} \int_{R_{+}^{n}} f^{p}(x) \frac{u^{a p}(x)}{v^{b p}(y)} K(u(x), v(y)) d x d y\right)^{1 / p} \\
& \times\left(\int_{R_{+}^{n}} \int_{R_{+}^{n}} g^{q}(y) \frac{v^{b q}(y)}{u^{a q}(x)} K(u(x), v(y)) d x d y\right)^{1 / q} \\
= & \left(\int_{R_{+}^{n}}(u(x))^{\frac{\alpha+n}{q}} f^{p}(x) \omega_{1}(x) d x\right)^{1 / p}\left(\int_{R_{+}^{n}}(v(y))^{\frac{\beta+n}{p}} g^{q}(y) \omega_{2}(y) d y\right)^{1 / q} \\
= & W_{1}^{1 / p} W_{2}^{1 / q}\left(\int_{R_{+}^{n}}(u(x))^{\frac{\alpha+n}{q}+\frac{\lambda_{1}}{\lambda_{2}}\left(\frac{\beta+n}{q}-n\right)} f^{p}(x) d x\right)^{1 / p} \\
& \times\left(\int_{R_{+}^{n}}(v(y))^{\frac{\beta+n}{p}+\frac{\lambda_{2}}{\lambda_{1}}\left(\frac{\alpha+n}{p}-n\right)} g^{q}(y) d y\right)^{1 / q} \\
= & W_{1}^{1 / p} W_{2}^{1 / q}\left(\int_{R_{+}^{n}}(u(x))^{\alpha} f^{p}(x) d x\right)^{1 / p}\left(\int_{R_{+}^{n}}(v(y))^{\beta} g^{q}(y) d y\right)^{1 / q} \\
= & W_{1}^{1 / p} W_{2}^{1 / q}\|f\|_{p, u^{\alpha}}\|g\|_{q, v^{\beta}} .
\end{aligned}
$$

Taking $M \geq W_{1}^{1 / p} W_{2}^{1 / q}$, we prove that (4) is valid.

Theorem 2 With regards to the assumption of Theorem 1, the best possible constant factor of (4) is $\inf M=W_{1}^{1 / p} W_{2}^{1 / q}$ when (4) holds true.

Proof We assume that (4) is valid. If there exists a positive number $M_{0}<W_{1}^{1 / p} W_{2}^{1 / q}$ such that (4) is still valid when replacing $M$ by $M_{0}$, then, $\forall f(x) \in L_{u^{\alpha}(x)}^{p}\left(R_{+}^{n}\right)$ and $g(y) \in L_{\nu^{\beta}(y)}^{p}\left(R_{+}^{n}\right)$, we have

$$
\int_{R_{+}^{n}} \int_{R_{+}^{n}} K(u(x), v(y)) f(x) g(y) d x d y \leq M_{0}\|f\|_{p, u^{\alpha}}\|g\|_{q, v^{\beta}} .
$$

Taking $\varepsilon>0$ and $\delta>0$ small enough and setting

$$
\begin{aligned}
& f(x)= \begin{cases}(u(x))^{\left(-\alpha-n-\left|\lambda_{1}\right| \varepsilon\right) / p}, & u(x)>\delta, \\
0, & 0<u(x) \leq \delta,\end{cases} \\
& g(y)= \begin{cases}(v(y))^{\left(-\beta-n+\left|\lambda_{2}\right| \varepsilon\right) / q}, & 0<v(y)<1, \\
0, & v(y) \geq 1,\end{cases}
\end{aligned}
$$


we have

$$
\begin{aligned}
& \|f\|_{p, u^{\alpha}}\|g\|_{q, v^{\beta}} \\
& =\left(\int_{\Omega(\delta<+\infty)}(u(x))^{-n-\left|\lambda_{1}\right| \varepsilon} d x\right)^{1 / p}\left(\int_{\Omega^{\prime}(0<1)}(v(y))^{-n+\left|\lambda_{2}\right| \varepsilon} d y\right)^{1 / q} \\
& =\frac{\Gamma^{n}\left(\frac{1}{\rho}\right)\left(\frac{1}{\delta^{1} \lambda_{1}^{|\varepsilon| \rho}}\right)^{1 / p}}{\left|\lambda_{1}\right|^{1 / p}\left|\lambda_{2}\right|^{1 / q} \rho^{n-1} \Gamma\left(\frac{n}{\rho}\right) \varepsilon}\left(\prod_{i=1}^{n} a_{i}^{-1 / \rho}\right)^{1 / p}\left(\prod_{i=1}^{n} b_{i}^{-1 / \rho}\right)^{1 / q} .
\end{aligned}
$$

And we have the following by using $\frac{n \lambda_{1}+\alpha \lambda_{2}}{p}=\frac{n \lambda_{2}+\beta \lambda_{1}}{q}$ :

$$
\begin{aligned}
\int_{R_{+}^{n}} & \int_{R_{+}^{n}} K(u(x), v(y)) f(x) g(y) d x d y \\
= & \int_{\Omega^{\prime}(0<1)}(v(y))^{\left(-\beta-n+\left|\lambda_{2}\right| \varepsilon\right) / q}\left(\int_{\Omega(\delta<+\infty)}(u(x))^{\left(-\alpha-n-\left|\lambda_{1}\right| \varepsilon\right) / p} K(u(x), v(y)) d x\right) d y \\
= & \int_{\Omega^{\prime}(0<1)}(v(y))^{\left(-\beta-n+\left|\lambda_{2}\right| \varepsilon\right) / q} \\
& \times\left(\int_{\Omega(\delta<+\infty)}(u(x))^{\frac{-\alpha-n-\left|\lambda_{1}\right| \varepsilon}{p}} K\left(u\left(v^{\frac{\lambda_{2}}{\lambda_{1}}}(y) x, 1\right)\right) d x\right) d y \\
= & \int_{\Omega^{\prime}(0<1)}(v(y))^{\left(-\beta-n+\left|\lambda_{2}\right| \varepsilon\right) / q}\left(\int_{\Omega\left(\delta \nu^{\frac{\lambda_{2}}{\lambda_{1}}}(y)<+\infty\right)}\left(v^{-\frac{\lambda_{2}}{\lambda_{1}}}(y) u(t)\right)^{\frac{-\alpha-n-\left|\lambda_{1}\right| \varepsilon}{p}}\right. \\
& \left.\times K(u(t), 1) v^{-\frac{n \lambda_{2}}{\lambda_{1}}}(y) d t\right) d y \\
= & \int_{\Omega^{\prime}(0<1)}(v(y))^{-n+\left|\lambda_{2}\right| \varepsilon}\left(\int_{\Omega\left(\delta v^{\frac{\lambda_{2}}{\lambda_{1}}}(y)<+\infty\right)}(u(t))^{\frac{-\alpha-n-\left|\lambda_{1}\right| \varepsilon}{p}} K(u(t), 1) d t\right) d y \\
\geq & \int_{\Omega^{\prime}(0<1)}(v(y))^{-n+\left|\lambda_{2}\right| \varepsilon} d y \int_{\Omega(\delta<+\infty)}(u(t))^{\frac{-\alpha-n-\left|\lambda_{1}\right| \varepsilon}{p}} K(u(t), 1) d t \\
= & \frac{\Gamma^{n}\left(\frac{1}{\rho}\right) \prod_{i=1}^{n} b_{i}^{-1 / \rho}}{\left|\lambda_{2}\right| \rho^{n-1} \Gamma\left(\frac{n}{\rho}\right) \varepsilon} \int_{\Omega(\delta<+\infty)}(u(t))^{\frac{-\alpha-n-\left|\lambda_{1}\right| \varepsilon}{p}} K(u(t), 1) d t .
\end{aligned}
$$

Combining (13), (14) and (15), we have

$$
\begin{aligned}
& \int_{\Omega(\delta<+\infty)} K(u(t), 1)(u(t))^{\frac{-\alpha-n-\left|\lambda_{1}\right| \varepsilon}{p}} d t \\
& \quad \leq M_{0}\left(\frac{1}{\left|\lambda_{1}\right|} \prod_{i=1}^{n} a_{i}^{-1 / \rho}\right)^{1 / p}\left(\frac{1}{\left|\lambda_{2}\right|} \prod_{i=1}^{n} b_{i}^{-1 / \rho}\right)^{1 / p}\left(\frac{1}{\delta^{\left|\lambda_{1}\right| \varepsilon / \rho}}\right)^{1 / p} .
\end{aligned}
$$

If we set

$$
\begin{aligned}
& f(x)= \begin{cases}(u(x))^{\left(-\alpha-n-\left|\lambda_{1}\right| \varepsilon\right) / p}, & 0<u(x)<1, \\
0, & u(x) \geq 1,\end{cases} \\
& g(y)= \begin{cases}(v(y))^{\left(-\beta-n+\left|\lambda_{2}\right| \varepsilon\right) / q}, & v(y)>\delta, \\
0, & 0<v(y) \leq \delta,\end{cases}
\end{aligned}
$$


then, in the same way, we have

$$
\begin{aligned}
& \int_{\Omega^{\prime}(\delta<+\infty)} K(1, v(t))(v(y))^{\left(-\beta-n+\left|\lambda_{2}\right| \varepsilon\right) / q} d t \\
& \quad \leq M_{0}\left(\frac{1}{\left|\lambda_{1}\right|} \prod_{i=1}^{n} a_{i}^{-1 / \rho}\right)^{1 / q}\left(\frac{1}{\left|\lambda_{2}\right|} \prod_{i=1}^{n} b_{i}^{-1 / \rho}\right)^{1 / q}\left(\frac{1}{\delta^{\left|\lambda_{2}\right| \varepsilon / \rho}}\right)^{1 / q} .
\end{aligned}
$$

Hence, by (16) and (17), we have

$$
\begin{aligned}
& \left(\int_{\Omega^{\prime}(\delta<+\infty)} K(1, v(t))(v(y))^{\left(-\beta-n+\left|\lambda_{2}\right| \varepsilon\right) / q} d t\right)^{1 / p} \\
& \quad \times\left(\int_{\Omega(\delta<+\infty)} K(u(t), 1)(u(t))^{\frac{-\alpha-n-\left|\lambda_{1}\right| \varepsilon}{p}} d t\right)^{1 / q} \\
& \quad \leq M_{0}\left(\frac{1}{\delta^{\left|\lambda_{2}\right| \varepsilon / \rho}}\right)^{1 /(p q)}\left(\frac{1}{\delta^{\left|\lambda_{1}\right| \varepsilon / \rho}}\right)^{1 /(p q)} .
\end{aligned}
$$

For $\varepsilon \rightarrow 0^{+}$, using Fatou's lemma, we obtain

$$
\left(\int_{\Omega^{\prime}(\delta<+\infty)} K(1, v(t))(v(y))^{-\frac{\beta+n}{q}} d t\right)^{\frac{1}{p}}\left(\int_{\Omega(\delta<+\infty)} K(u(t), 1)(u(t))^{-\frac{\alpha+n}{p}} d t\right)^{\frac{1}{q}} \leq M_{0}
$$

and then it follows that, for $\delta \rightarrow 0^{+}$,

$$
W_{1}^{\frac{1}{p}} W_{2}^{\frac{1}{q}}=\left(\int_{R_{+}^{n}}(v(y))^{-\frac{\beta+n}{q}} K(1, v(t)) d t\right)^{\frac{1}{p}}\left(\int_{R_{+}^{n}}(u(t))^{-\frac{\alpha+n}{p}} K(u(t), 1) d t\right)^{\frac{1}{q}} \leq M_{0} .
$$

This is a contradiction, which leads to the fact that $W_{1}^{1 / p} W_{2}^{1 / q}$ is the best possible constant factor of (4).

\section{Application in the operator theory}

For $\gamma=\beta(1-p)$, there is $-\frac{\beta+n}{q}=\frac{\gamma+n}{p}-n$, and it follows that $\frac{n \lambda_{1}+\alpha \lambda_{2}}{p}=\frac{n \lambda_{2}+\beta \lambda_{1}}{q}$ is equivalent to $\lambda_{1}(n+\gamma)+\lambda_{2}(n+\alpha)=\lambda_{2} n p$. In view of the fact that (1) is equivalent to (3), by Theorems 1-2, we have the following.

Theorem 3 Suppose that $n \geq 1, p>1, \rho>0, \alpha, \gamma \in R, \lambda_{1} \lambda_{2}>0, a_{i}>0, b_{i}>0, u(x)=$ $\left(\sum_{i=1}^{\infty} a_{i} x_{i}^{\rho}\right)^{1 / \rho}, v(y)=\left(\sum_{i=1}^{\infty} b_{i} y_{i}^{\rho}\right)^{1 / \rho}, K(u(x), v(y))=G\left(u^{\lambda_{1}}(x) v^{\lambda_{2}}(y)\right)$ is a non-negative measurable function, the operator $T$ is defined by (2),

$$
\begin{aligned}
& 0<\tilde{W}_{1}=\int_{R_{+}^{n}}(v(t))^{\frac{\gamma+n}{p}-n} K(1, v(t)) d t<\infty, \\
& 0<\tilde{W}_{2}=\int_{R_{+}^{n}}(u(t))^{-\frac{\alpha+n}{p}} K(u(t), 1) d t<\infty,
\end{aligned}
$$

and for $a=0, b=1($ or $a=1, b=+\infty)$,

$$
\int_{\Omega^{\prime}(a<b)}(v(t))^{\frac{\gamma+n}{p}-n} K(1, v(t)) d t>0, \quad \int_{\Omega^{\prime}(a<b)}(u(t))^{-\frac{\alpha+n}{p}} K(u(t), 1) d t>0
$$


then we have the following:

(i) T is a bounded operator from $L_{u^{\alpha}}^{p}\left(R_{+}^{n}\right)$ to $L_{v^{\gamma}}^{p}\left(R_{+}^{n}\right)$ if and only if the equality $\lambda_{1}(n+\gamma)+$ $\lambda_{2}(n+\alpha)=\lambda_{2} n p$ is valid.

(ii) If the operator $T$ is a bounded operator from $L_{u^{\alpha}}^{p}\left(R_{+}^{n}\right)$ to $L_{\nu \gamma}^{p}\left(R_{+}^{n}\right)$, then we obtain the norm of the operator $T$ as follows:

$$
\|T\|:=\sup _{f \in L_{u^{\alpha}}^{p}\left(R_{+}^{n}\right)} \frac{\|T(f)\|_{p, v^{\gamma}}}{\|f\|_{p, u^{\rho}}}=\tilde{W}_{1}^{\frac{1}{p}} \tilde{W}_{2}^{\frac{1}{q}}
$$

Taking $\alpha=\gamma=0$ in Theorem 3, we have the result as follows.

Corollary 1 Suppose that $n \geq 1, p>1, \rho>0, \lambda_{1} \lambda_{2}>0, a_{i}>0, b_{i}>0(i=1, \ldots, n), u(x)=$ $\left(\sum_{i=1}^{\infty} a_{i} x_{i}^{\rho}\right)^{1 / \rho}, v(y)=\left(\sum_{i=1}^{\infty} b_{i} y_{i}^{\rho}\right)^{1 / \rho}, K(u(x), v(y))=G\left(u^{\lambda_{1}}(x) v^{\lambda_{2}}(y)\right)$ is a non-negative measurable function, the operator $T$ is defined by (2),

$$
\begin{aligned}
& 0<\tilde{W}_{1}=\int_{R_{+}^{n}}(v(t))^{\frac{n}{p}-n} K(1, v(t)) d t<\infty, \\
& 0<\tilde{W}_{2}=\int_{R_{+}^{n}}(u(t))^{-\frac{n}{p}} K(u(t), 1) d t<\infty,
\end{aligned}
$$

and for $a=0, b=1($ or $a=1, b=+\infty)$,

$$
\int_{\Omega^{\prime}(a<b)}(v(t))^{\frac{n}{p}-n} K(1, v(t)) d t>0, \quad \int_{\Omega^{\prime}(a<b)}(u(t))^{-\frac{n}{p}} K(u(t), 1) d t>0,
$$

then we have the following:

(i) $T$ is a bounded operator in $L^{p}\left(R_{+}^{n}\right)$ if and only if $\lambda_{1}=(p-1) \lambda_{2}$.

(ii) If the operator $T$ is a bounded operator in $L^{p}\left(R_{+}^{n}\right)$, then the norm of the operator $T$ is

$$
\|T\|=\tilde{W}_{1}^{\frac{1}{p}} \tilde{W}_{2}^{\frac{1}{q}} .
$$

Theorem 4 Suppose that $n \geq 1, p>1, \frac{1}{p}+\frac{1}{q}=1, \rho>0, \lambda_{1}, \lambda_{2}>0, a_{i}>0, b_{i}>0(i=1, \ldots, n)$, $b>\frac{n}{\lambda_{2} p}, a>b-\frac{n}{\lambda_{2} p}, u(x)=\left(\sum_{i=1}^{\infty} a_{i} x_{i}^{\rho}\right)^{\frac{1}{\rho}}, v(y)=\left(\sum_{i=1}^{\infty} b_{i} y_{i}^{\rho}\right)^{\frac{1}{\rho}}$, the operator $T$ is defined $b y$

$$
T(f)(y)=\int_{R_{+}^{n}} \frac{\left(u^{\lambda_{1}}(x) v^{\lambda_{2}}(y)\right)^{b}}{\left(1+u^{\lambda_{1}}(x) v^{\lambda_{2}}(y)\right)^{a}} f(x) d x, \quad y \in R_{+}^{n},
$$

then we have the following:

(i) $T$ is a bounded operator in $L^{p}\left(R_{+}^{n}\right)$ if and only if $\frac{\lambda_{1}}{p}=\frac{\lambda_{2}}{q}$.

(ii) If the operator $T$ is a bounded operator in $L^{p}\left(R_{+}^{n}\right)$, then the norm of the operator $T$ is as follows:

$$
\|T\|=\frac{\Gamma^{n}\left(\frac{1}{\rho}\right)}{\lambda_{1}^{\frac{1}{q}} \lambda_{2}^{\frac{1}{p}} \rho^{n-1} \Gamma\left(\frac{n}{\rho}\right) \Gamma(a)}\left(\prod_{i=1}^{n} a_{i}^{-\frac{1}{\rho}}\right)^{\frac{1}{q}}\left(\prod_{i=1}^{n} b_{i}^{-\frac{1}{\rho}}\right)^{\frac{1}{p}} \Gamma\left(b-\frac{n}{\lambda_{2} p}\right) \Gamma\left(a-b+\frac{n}{\lambda_{2} p}\right) .
$$


Proof (ii) In view of $\frac{\lambda_{1}}{p}=\frac{\lambda_{2}}{q}$, we have the following by using Lemma 2:

$$
\begin{aligned}
& \tilde{W}_{1}=\int_{R_{+}^{n}}(v(t))^{-\frac{n}{q}} K(1, v(t)) d t \\
& =\int_{R_{+}^{n}}\left(\sum_{i=1}^{n} b_{i} t_{i}^{\rho}\right)^{\frac{\lambda_{2} b}{\rho}-\frac{n}{q \rho}} \frac{1}{\left[1+\left(\sum_{i=1}^{n} b_{i} t_{i}^{\rho}\right)^{\lambda_{2} / \rho}\right]^{a}} d t \\
& =\prod_{i=1}^{n} b_{i}^{-\frac{1}{\rho}} \int_{R_{+}^{n}}\left(\sum_{i=1}^{n} x_{i}^{\rho}\right)^{\frac{\lambda_{2} b}{\rho}-\frac{n}{q \rho}} \frac{1}{\left[1+\left(\sum_{i=1}^{n} x_{i}^{\rho}\right)^{\lambda_{2} / \rho}\right]^{a}} d t \\
& =\prod_{i=1}^{n} b_{i}^{-\frac{1}{\rho}} \lim _{r \rightarrow \infty} \int \cdots \int_{x_{i}>0 ; x_{1}^{p}+\cdots+x_{n}^{p} \leq r^{p}} \frac{r^{\lambda_{2} b-n / q}}{\left[1+r^{\lambda_{2}}\left(\sum_{i=1}^{n}\left(\frac{x_{i}}{r}\right)^{\rho}\right)^{\lambda_{2} / \rho}\right]^{a}} \\
& \times\left(\sum_{i=1}^{n}\left(\frac{x_{i}}{r}\right)^{\rho}\right)^{\frac{\lambda_{2} b}{\rho}-\frac{n}{q \rho}} x_{1}^{1-1} \cdots x_{n}^{1-1} d x_{1} \cdots d x_{n} \\
& =\prod_{i=1}^{n} b_{i}^{-\frac{1}{\rho}} \lim _{r \rightarrow \infty} r^{\lambda_{2} b-\frac{n}{q}} \frac{r^{n} \Gamma^{n}\left(\frac{1}{\rho}\right)}{\rho^{n} \Gamma\left(\frac{n}{\rho}\right)} \int_{0}^{1} \frac{u^{\frac{\lambda_{2} b}{\rho}-\frac{n}{q \rho}+\frac{n}{\rho}-1}}{\left(1+r^{\lambda_{2}} u^{\lambda_{2} / \rho}\right)^{a}} d u \\
& =\prod_{i=1}^{n} b_{i}^{-\frac{1}{\rho}} \lim _{r \rightarrow \infty} \frac{\Gamma^{n}\left(\frac{1}{\rho}\right)}{\lambda_{2} \rho^{n-1} \Gamma\left(\frac{n}{\rho}\right)} \int_{0}^{r^{\lambda_{2}}} \frac{1}{(1+t)^{a}} t^{b-\frac{n}{\lambda_{2} p}-1} d t \\
& =\frac{\Gamma^{n}\left(\frac{1}{\rho}\right)}{\lambda_{2} \rho^{n-1} \Gamma\left(\frac{n}{\rho}\right)} \prod_{i=1}^{n} b_{i}^{-\frac{1}{\rho}} \int_{0}^{+\infty} \frac{1}{(1+t)^{a}} t^{b-\frac{n}{\lambda_{2} p}-1} d t \\
& =\frac{\Gamma^{n}\left(\frac{1}{\rho}\right)}{\lambda_{2} \rho^{n-1} \Gamma\left(\frac{n}{\rho}\right)} \prod_{i=1}^{n} b_{i}^{-\frac{1}{\rho}} B\left(b-\frac{n}{\lambda_{2} p}, a-\left(b-\frac{n}{\lambda_{2} p}\right)\right) \\
& =\frac{\Gamma^{n}\left(\frac{1}{\rho}\right)}{\lambda_{2} \rho^{n-1} \Gamma\left(\frac{n}{\rho}\right) \Gamma(a)} \prod_{i=1}^{n} b_{i}^{-\frac{1}{\rho}} \Gamma\left(b-\frac{n}{\lambda_{2} p}\right) \Gamma\left(a-b+\frac{n}{\lambda_{2} p}\right) .
\end{aligned}
$$

In the same way, we still have the following:

$$
\begin{aligned}
\tilde{W}_{2} & =\int_{R_{+}^{n}}[u(t)]^{-\frac{n}{p}} K(u(t), 1) d t \\
& =\frac{\Gamma^{n}\left(\frac{1}{\rho}\right)}{\lambda_{1} \rho^{n-1} \Gamma\left(\frac{n}{\rho}\right) \Gamma(a)} \prod_{i=1}^{n} a_{i}^{-\frac{1}{\rho}} \Gamma\left(b-\frac{n}{\lambda_{1} q}\right) \Gamma\left(a-b+\frac{n}{\lambda_{1} q}\right) \\
& =\frac{\Gamma^{n}\left(\frac{1}{\rho}\right)}{\lambda_{1} \rho^{n-1} \Gamma\left(\frac{n}{\rho}\right) \Gamma(a)} \prod_{i=1}^{n} a_{i}^{-\frac{1}{\rho}} \Gamma\left(b-\frac{n}{\lambda_{2} p}\right) \Gamma\left(a-b+\frac{n}{\lambda_{2} p}\right) .
\end{aligned}
$$

It follows that

$$
\tilde{W}_{1}^{\frac{1}{p}} \tilde{W}_{2}^{\frac{1}{q}}=\frac{\Gamma^{n}\left(\frac{1}{\rho}\right)}{\lambda_{1}^{\frac{1}{q}} \lambda_{2}^{\frac{1}{p}} \rho^{n-1} \Gamma\left(\frac{n}{\rho}\right) \Gamma(a)}\left(\prod_{i=1}^{n} a_{i}^{-\frac{1}{\rho}}\right)^{\frac{1}{q}}\left(\prod_{i=1}^{n} b_{i}^{-\frac{1}{\rho}}\right)^{\frac{1}{p}} \Gamma\left(b-\frac{n}{\lambda_{2} p}\right) \Gamma\left(a-b+\frac{n}{\lambda_{2} p}\right) .
$$

Hence, we prove that (ii) is valid by Corollary 1 . 


\section{Conclusions}

In this paper, by using the methods and techniques of real analysis, the sufficient and necessary conditions for the existence of the Hilbert-type multiple integral inequality with the kernel $K(u(x), v(y))=G\left(u^{\lambda_{1}}(x) v^{\lambda_{2}}(y)\right)$ and the best possible constant factor are discussed in Theorems 1-2. Furthermore, its application in the operator theory is considered in Theorems 3-4. The method of real analysis is very important as itis the key to prove the equivalent inequalities with the best possible constant factor. The lemmas and theorems provide an extensive account of this type of inequalities.

\section{Acknowledgements}

This work is supported by the National Nature Science Foundation of China (No. 11401113) and Training Plan Fund of Outstanding Young Teachers of Higher Learning Institutions of Guangdong Province of China (No. YQ2015122). We are grateful for this help.

\section{Competing interests}

The authors declare that they have no competing interests.

\section{Authors' contributions}

YH carried out the mathematical studies, participated in the sequence alignment and drafted the manuscript. QH, BY and $J L$ participated in the design of the study and performed the numerical analysis. All authors read and approved the final manuscript.

\section{Author details}

'School of Mathematics and Statistics, Guangdong University of Finance and Economics, Guangzhou, Guangdong 510320, P.R. China. '2Department of Mathematics, Guangdong University of Education, Guangzhou, Guangdong 510303, P.R. China.

\section{Publisher's Note}

Springer Nature remains neutral with regard to jurisdictional claims in published maps and institutional affiliations.

Received: 31 October 2017 Accepted: 3 December 2017 Published online: 28 December 2017

\section{References}

1. Hong, Y: A Hilbert-type integral inequality with quasi-homogeneous kernel and several functions. Acta Math. Sin., Chin. Ser. 57(5), 833-840 (2014)

2. Rassias, MT, Yang, B: On a Hardy-Hilbert-type inequality with a general homogeneous kernel. Int. J. Nonlinear Anal. Appl. 7(1), 249-269 (2016)

3. Chen, Q, Shi, Y, Yang, B: A relation between two simple Hardy-Mulholland-type inequalities with parameters. J. Inequal. Appl. 2016, 75 (2016)

4. Hong, $Y$, Wen, $Y$ : A necessary and sufficient condition for a Hilbert type series inequality with homogeneous kernel to have the best constant factor. Chin. Ann. Math. 2016, 329-336 (2016)

5. Yang, B, Chen, Q: On a more accurate Hardy-Mulholland-type inequality. J. Inequal. Appl. 2016, 82 (2016)

6. Gao, M, Yang, B: On the extended Hilbert's inequality. Proc. Am. Math. Soc. 126(3), 751-759 (1998)

7. Yang, B: On a more accurate multidimensional Hilbert-type inequality with parameters. Math. Inequal. Appl. 18, 429-441 (2015)

8. Xin, D, Yang, B, Chen, Q: A discrete Hilbert-type inequality in the whole plane. J. Inequal. Appl. 2016, 133 (2016)

9. Kuang, J: Applied Inequalities. Shangdong Science and Technology Press, Jinan (2004)

10. Kuang, J, Debnath, L: On Hilbert's type integral inequalities on the weighted Orlicz spaces. Pac. J. Appl. Math. 1, 89-98 (2008)

11. Yang, B, Chen, Q: On a Hardy-Hilbert-type inequality with parameters. J. Inequal. Appl. 2015, 339 (2015)

12. Yang, B, Chen, Q: A new extension of Hardy-Hilbert's inequality in the whole plane. J. Funct. Spaces 2016, Article ID $9197476(2016)$

13. Zeng, Z, Xie, Z: On a new Hilbert-type integral inequality with the homogeneous kernel of degree 0 and the integral in whole plane. J. Inequal. Appl. 2010, Article ID 256796 (2010)

14. Huang, Q, Yang, B: On a multiple Hilbert-type integral operator and applications. J. Inequal. Appl. 2009, Article ID 192197 (2009)

15. Fichtinggoloz, GM: A Course in Differential and Integral Calculus. People Education Press, Beijing (1975) 